\title{
2021 Canadian Urological Association (CUA)-Canadian Uro Oncology Group (CUOG) guideline: Management of castration-resistant prostate cancer (CRPC)
}

\author{
Fred Saad, $M D^{1}$; Armen Aprikian, $M D^{2}$; Antonio Finelli, $M D^{3}$; Neil E. Fleshner, $M D^{3}$; Martin Gleave, $M D^{4}$;

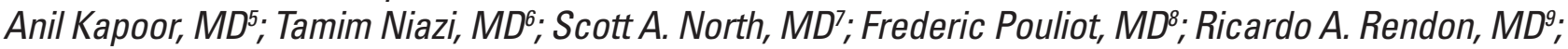 \\ Bobby Shayegan, MD'; Srikala S. Sridhar, MD ${ }^{10}$; Alan So, MD'; Nawaid Usmani, MD ${ }^{11}$; Eric Vigneault, MD ${ }^{12}$; \\ Kim N. Chi, MD's
}

'Centre Hospitalier de I'Université de Montréal, Montreal, QC, Canada; ${ }^{2}$ McGill University Health Center, Montreal, QC, Canada; ${ }^{3}$ Division of Urology, University of Toronto, Toronto, ON, Canada; ${ }^{4}$ Department of Urological Sciences, University of British Columbia, Vancouver, BC, Canada; ${ }^{5}$ Department of Surgery (Urology) and Oncology, McMaster University, Hamilton, ON, Canada; ${ }^{6}$ Jewish General Hospital, Montreal, QC, Canada; ' Department of Medical Oncology, University of Alberta, Edmonton, AB, Canada; ${ }^{8}$ CHU de Quebec, Université Laval, Quebec City, QC, Canada; ${ }^{9}$ Department of Urology, Dalhousie University, Halifax, NS, Canada; ${ }^{10}$ Division of Medical Oncology, University of Toronto, Toronto, ON, Canada; "Department of Oncology, Division of Radiation Oncology, University of Alberta, Edmonton, AB, Canada; ' 2 Department of Radiation Oncology, CHU de Quebec, Université Laval, Quebec City, QC, Canada; ${ }^{13} \mathrm{BC}$ Cancer Agency, Vancouver, BC, Canada

Cite as: Saad F, Aprikian A, Finelli A, et al. 2021 Canadian Urological Association (CUA)-Canadian Uro Oncology Group (CUOG) guideline: Management of castration-resistant prostate cancer (CRPC). Can Urol Assoc J 2021;15(2):E81-9. http://dx.doi.org/10.5489/cuaj.7074

This is an update of CUA guideline previously published online June 25, 2019. A brief summary of the changes is available at cuaj.ca

MEDLINE search of the English language and conference proceedings were used to produce the present document. Wherever Level 1 evidence is lacking, the guideline attempts to provide expert opinion to aid in the management of patients.

Levels of evidence and grades of recommendation employ the International Consultation on Urologic Disease (ICUD)/ WHO modified Oxford Center for Evidence-Based Medicine grading system. Based on a modified GRADE methodology, the strength of each recommendation is represented by the words STRONG or WEAK.

\section{Introduction}

Castration-resistant prostate cancer (CRPC) is defined by disease progression despite castrate levels of testosterone and may present as either a continuous rise in serum prostatespecific antigen (PSA) levels, the progression of pre-existing disease, and/or the appearance of new metastases.

Advanced prostate cancer has been known under a few names over the years, including hormone-resistant prostate cancer (HRPC) and androgen-insensitive prostate cancer (AIPC). Most recently, the terms castration-resistant prostate cancer or castration-recurrent prostate cancer were introduced with the realization that extra-testicular androgen production plays a significant role in the resistance of prostate cancer cells to medical or surgical castration therapy. ${ }^{1}$

In their second publication, the Prostate Cancer Working Group defined CRPC as a continuum on the basis of whether metastases are detectable (clinically or by imaging) and whether the serum testosterone is in the castrate range by surgical orchidectomy or medical therapy. ${ }^{2}$ This definition creates a clinical-states model, where patients can be classified. The rising PSA states (castrate and non-castrate) signify that no detectable (measurable or non-measurable) disease has ever been found. The clinical metastases states (castrate and non-castrate) signify that disease was detectable at some point in the past, regardless of whether it is detectable now. ${ }^{3}$

Prognosis is associated with several factors that go beyond PSA levels. These include performance status, presence of visceral metastases, presence of bone pain, extent of disease on bone scan, and serum lactate dehydrogenase and alkaline phosphatase levels. Bone metastases will occur in 90\% of men with CRPC and can produce significant morbidity, including pain, pathological fractures, spinal cord compression, and bone marrow failure. Paraneoplastic effects, including anemia, weight loss, fatigue, hypercoagulability, and increased susceptibility to infection, are also common.

CRPC includes patients without metastases or symptoms with rising PSA levels despite androgen deprivation therapy (ADT) to patients with metastases and significant debilitation due to cancer symptoms. 


\section{Management of CRPC}

\section{ADT and first-generation androgen receptor antagonists}

Because the androgen receptor remains active in most patients who have developed castration-resistant disease, it is recommended that ADT be continued for the remainder of a patient's life (Level 3, Weak recommendation).

In patients who develop CRPC, the addition or change of first-generation androgen receptor antagonists may be considered (Level 3, Weak recommendation).

To date, no study using first-generation androgen receptor antagonists, when introduced in the CRPC setting, has shown survival benefits; most trials have been small, were not designed to evaluate overall survival (OS), and were heavily confounded by future treatments used. In patients treated with luteinizing hormone-releasing hormone (LHRH) agonist/antagonist monotherapy or those who have had an orchidectomy, the addition of androgen receptor antagonists, such as bicalutamide, can offer modest PSA responses that are short-lived in $30-35 \%$ of patients. ${ }^{4}$

For patients who have undergone total androgen blockade $(T A B)$, the anti-androgen (AA) should be discontinued to test for an anti-androgen withdrawal response (AAWD). Changing AA or using corticosteroids with or without ketoconazole have been noted to cause transient PSA reductions in about $30 \%$ of patients but have not been shown to improve any of the clinically meaningful outcome measures.

\section{Detection of metastases and imaging in untreated patients}

For patients who progress on ADT without evidence of distant metastases, it is suggested to screen for bone metastases with bone scans and monitor for lymph node and visceral metastases/progression with imaging of the abdomen/pelvis and chest.

Patients with a rapid PSADT ( $<10$ months) or elevated PSA levels $(>20)$ are at high risk for developing metastases earlier. ${ }^{3}$ Imaging in these patients should be performed every 3-6 months. Patients with a slower PSADT (>10 months) should be screened every 6-12 months (Expert opinion). Imaging techniques most commonly used include nuclear bone scans and abdominal/pelvic CT and chest X-ray. The role of positronemission tomography (PET), such as prostate specific membrane antigen (PSMA)-PET are still unclear and the benefits unknown.

If and when metastases are detected, patients should be treated according to guidelines for metastatic CRPC (mCRPC). How patients are treated in the MCRPC state will depend on what they received prior to becoming $\mathrm{MCRPC}$.

Non-metastatic CRPC (nmCRPC)

Men with high-risk nmCRPC, defined as a PSA doubling time (PSADT) of less than 10 months, with an estimated life expectancy of greater than five years should be offered apalutamide, enzalutamide, or darolutamide (Level 1, Strong recommendation).

Until 2018, there was no standard of care and no approved regimen for the nmCRPC state. The risk of progression to clinical metastases or death is linked to PSADT. PSADT of less than 10 months has been correlated with worse outcome and has been used in recent clinical trials as the definition for highrisk nmCRPC. Patients in these studies were randomized to treatment + ADT vs. placebo + ADT until the appearance of metastases on conventional imaging (bone scan and computed tomography $[\mathrm{CT}] /$ magnetic resonance imaging [MRI] of the abdomen/chest). The three studies used second-generation androgen receptor (AR)-targeted therapies (apalutamide, enzalutamide, and darolutamide) and reported similar results in significantly improving the primary endpoint of metastasesfree survival (MFS). At the first report of results for the three trials, median OS, a secondary endpoint, was not reached but at interim analysis, there was a non-significant improvement in OS for men receiving the AR-targeted therapies. ${ }^{5-7}$

At final analysis, the three agents demonstrated statistically significant improvements in OS. The phase 3 studies have led to Health Canada approvals of apalutamide, enzalutamide, and darolutamide for the treatment of high-risk nmCRPC.

\section{Summary of results}

\section{Apalutamide}

Apalutamide is a second-generation AR ligand-binding domain inhibitor. This agent was tested in combination with standard ADT in patients with nmCRPC at high risk for progression (PSADT of $\leq 10$ months). ${ }^{5}$ The median MFS was 40.5 months with apalutamide and 16.2 months with placebo (hazard ratio [HR] for metastasis or death $0.28 ; 95 \%$ confidence interval $[\mathrm{Cl}]$ 0.23-0.35; $\mathrm{p}<0.001)$. Secondary endpoints analyzed, including progression-free survival (PFS) (local and distant), time to PSA progression, and time to subsequent therapy, were all statistically significantly improved. ${ }^{5}$ Although more adverse events were reported in patients receiving ADT + apalutamide vs. ADT + placebo, patient-reported health-related quality of life was similar between both groups. ${ }^{8}$

At final survival analysis, median followup time was 52.0 months. Median treatment duration was 32.9 months for apalutamide and 11.5 months for the placebo group. Median OS was significantly longer with apalutamide + ADT compared to placebo + ADT (73.9 months vs. 59.9 months, respectively; HR $0.784 ; p=0.0161$ ) The trial regimen was discontinued in $42.7 \%$ of the treatment group and $73.9 \%$ of the placebo group due to progressive disease, and $15.2 \%$ vs. $8.4 \%$ due to adverse events. The survival benefit was observed even though more than $85 \%$ of the patients 
in the placebo group received subsequent treatment. The SPARTAN trial concluded that apalutamide reduced the risk of metastasis or death and the MFS and OS benefit was consistent across all subgroups, including all age groups, local or regional nodal disease, and those with shorter or longer PSADT. ${ }^{9}$

\section{Enzalutamide}

Enzalutamide is a second-generation AR ligand-binding domain inhibitor. This agent was tested in combination with standard ADT in patients with nmCRPC at high risk for progression (PSADT of $\leq 10$ months). ${ }^{6}$ The median MFS was 36.6 months with enzalutamide and 14.7 months with placebo (HR for metastasis or death $0.29 ; 95 \% \mathrm{Cl} 0.24$ $0.35 ; \mathrm{p}<0.001)$. Secondary endpoints analyzed, including PFS (local and distant), time to PSA progression, and time to subsequent therapy, were all statistically significantly improved. Although more adverse events were reported in patients receiving ADT + enzalutamide vs. ADT + placebo, patient-reported health-related quality of life was similar between both groups. ${ }^{10}$

At final analysis, median follow-up was 48 months. At the time of cutoff, $31 \%$ of patients in the enzalutamide cohort and $38 \%$ of patients within the placebo group had died. In the enzalutamide cohort, $19 \%$ of deaths were from prostate cancer and $12 \%$ were not from prostate cancer. In the placebo group, $29 \%$ were from prostate cancer and 9\% were not from prostate cancer. Median OS was 67 months $(95 \% \mathrm{Cl}$ 64-not reached) in the enzalutamide group and 56.3 months (95\% Cl 54.4-63.0) in the placebo group. Enzalutamide + ADT was associated a $27 \%$ lower risk of death than placebo + ADT (HR 0.73; 95\% Cl 0.61-0.89; $\mathrm{p}=0.001) .{ }^{11}$

\section{Darolutamide}

Daroutamide is a second-generation AR ligand-binding domain inhibitor. This agent was tested in combination with standard ADT in patients with nmCRPC at high risk for progression (PSADT of $\leq 10$ months). ${ }^{7}$ The median MFS was 40.4 months with darolutamide and 18.4 months with placebo (HR for metastasis or death $0.41 ; 95 \% \mathrm{Cl} 0.34-0.50 ; p<0.001$ ). Secondary endpoints analyzed, including PFS (local and distant), time to PSA progression, and time to subsequent therapy, were all statistically significantly improved.? Although more adverse events were reported in patients receiving ADT + darolutamide vs. ADT + placebo, patient-reported healthrelated quality of life was similar between both groups. ${ }^{\text {? }}$

Final analysis was conducted after 254 deaths were observed $(15.5 \%$ of darolutamide group and $19.1 \%$ of placebo control group). Darolutamide had a statistically significant $31 \%$ reduction in the risk of death. After a median

\section{Guideline statements regarding nmCRPC}

1. ADT should be maintained in the nmCRPC state. First-generation androgen receptor antagonists (i.e., bicalutamide, flutamide, etc.) should be discontinued if patients are receiving these agents (Level 3, Weak recommendation).

2. Men with high-risk nmCRPC, defined as a PSADT $<10$ months, with an estimated life expectancy of greater than five years should be offered apalutamide, enzalutamide, or darolutamide (Level 1, Strong recommendation).

3. In men with high-risk nmCRPC who are felt to be unsuitable or refuse approved therapies, observation or use of first-generation androgen receptor antagonists may be attempted (Level 3, Weak recommendation).

4. For men with nmCRPC who are not considered high-risk, observation or secondary hormonal treatments may be attempted (Level 3, Weak recommendation).

5. Patients who are untreated for nmCRPC should be followed with regular imaging every 6-12 months depending on PSADT (Level 3, Weak recommendation).

followup time of 29 months, the median survival rate at three years was $83 \%$ in the darolutamide cohort and $77 \%$ in the placebo group ( $\mathrm{HR} 0.69 ; 95 \% \mathrm{Cl} 0.53-0.88 ; \mathrm{p}=0.003)$. The survival benefit was observed even though more than half of the patients in the placebo group received subsequent darolutamide treatment. ${ }^{12}$

Apalutamide, enzalutamide, and darolutamide have received Health Canada approval for use in high-risk nmCRPC.

\section{Treatment of $\mathrm{mCRPC}$}

Since mCRPC is generally associated with a high risk of morbidity and cancer-related mortality, patients with mCRPC detected on conventional imaging should be considered for systemic therapy with demonstrated survival benefits. Patients with mCRPC should optimally receive multidisciplinary care to maximize survival and quality of life. Because any treatment for advanced disease remains non-curative, patients with advanced prostate cancer should be encouraged to participate in clinical trials. 
Saad et al

\section{AR signaling therapeutic options}

In men with CRPC, phase 3 clinical trials have evaluated the role of abiraterone acetate and enzalutamide in both the chemo-naive and post-chemotherapy settings.

\section{Abiraterone acetate}

Abiraterone acetate is a potent and irreversible inhibitor of CYP-17, a critical enzyme in androgen biosynthesis.

In the chemo-naive setting: Abiraterone acetate $\mathbf{1 0 0 0}$ $\mathrm{mg}$ /day plus prednisone $5 \mathrm{mg}$ twice daily is recommended for first-line therapy for asymptomatic or minimally symptomatic mCRPC (Level 1, Strong recommendation).

In asymptomatic or minimally symptomatic patients (defined as pain that is relieved by acetaminophen or a nonsteroidal anti-inflammatory) without visceral metastases, abiraterone acetate significantly improved radiographic PFS (16.5 vs. 8.3 months) (HR 0.53; 95\% Cl 0.45-0.62; $p<0.001$ ) and had a statistically significant 4.4-month improvement in OS (HR 0.81; $p=0.0033){ }^{13,14}$ Abiraterone also significantly delayed time to pain progression, time to chemotherapy initiation, time to opiate initiation, and deterioration of the Eastern Cooperative Oncology Group (ECOG) performance status.

In the post-docetaxel setting: Abiraterone acetate $\mathbf{1 0 0 0}$ $\mathrm{mg}$ per day plus prednisone $\mathbf{5} \mathrm{mg}$ twice daily is recommended in patients progressing on or after docetaxel-based chemotherapy (Level 1, Strong recommendation).

In the post-docetaxel setting, abiraterone-prednisone compared to placebo-prednisone significantly prolonged median OS by 4.6 months (15.8 vs. 11.2 months; HR 0.74; $\mathrm{p}=0.0001$ ) in patients with mCRPC who had progressed after docetaxel treatment. Moreover, all secondary endpoints provided support for the superiority of abiraterone over placebo: median time to PSA progression (8.5 vs. 6.6 months; HR 0.63; $p<0.0001$ ), radiographic PFS (5.6 vs. 3.6 months; HR 0.66; $\mathrm{p}<0.0001)$, confirmed PSA response rate defined as $\geq 50 \%$ reduction in PSA from the pretreatment baseline PSA ( $29 \%$ vs. $5.5 \% ; p<0.0001)$, and objective response by Response Evaluation Criteria in Solid Tumors (RECIST) $(14.8 \%$ vs. $3.3 \% ; p<0.0001) .{ }^{15}$

\section{Enzalutamide}

Enzalutamide is a potent multi-targeted androgen signalling pathway inhibitor.

In the chemo-naive setting: Enzalutamide $160 \mathrm{mg}$ per day is recommended as first-line therapy for asymptomatic or minimally symptomatic mCRPC (Level 1, Strong recommendation).

In asymptomatic or minimally symptomatic patients (defined as pain that is relieved by acetaminophen or a nonsteroidal anti-inflammatory), enzalutamide decreased the risk of radiographic progression or death by $81 \%$ (HR $0.19 ; 95 \%$ $\mathrm{Cl} 0.15-0.23 ; \mathrm{p}<0.001)$ and the risk of death by $29 \%$ (HR $0.71 ; 95 \% \mathrm{Cl} 0.60-0.84 ; \mathrm{p}<0.001)$ as compared to placebo. The benefit of enzalutamide was demonstrated for all secondary endpoints, including time to initiation of cytotoxic chemotherapy, time to first skeletal-related event (SRE), best overall soft tissue response ( $59 \%$ vs. $5 \%$; $p<0.001)$, time to PSA progression (HR 0.17; $<<0.001$ ), and $\geq 50 \%$ PSA decline rate $(78 \%$ vs. $4 \%$; $<0.001)$. Enzalutamide also significantly delayed time to pain progression, time to opiate initiation, and deterioration of the ECOG performance status. ${ }^{16,17}$

In the post-docetaxel setting: Enzalutamide $160 \mathrm{mg}$ per day is recommended in patients progressing on or after docetaxelbased chemotherapy (Level 1, Strong recommendation).

In patients previously treated with docetaxel, the trial compared enzalutamide and placebo. The study demonstrated a significant advantage in OS of 4.8 months (18.4 vs. 13.6 months; HR $0.62 ; \mathrm{p}<0.0001$ ) and in all secondary endpoints, including confirmed PSA response rate $(54 \%$ vs. $2 \% ; p<0.001)$, soft-tissue response rate $(29 \%$ vs. $4 \%$; $\mathrm{p}<0.001$ ), time to PSA progression (8.3 vs. 3.0 months; HR $0.25 ; \mathrm{p}<0.001)$, radiographic PFS (8.3 vs. 2.9 months; HR $0.40 ; p<0.001)$, and the time to the first SRE ( 16.7 vs. 13.3 months; HR 0.69; $\mathrm{p}<0.001) .^{18}$

NOTE: The studies in the chemo-naive setting did not include patients with moderate or severe symptoms; however, abiraterone and enzalutamide may be potential therapeutic options in patients who are deemed chemotherapyineligible or refuse chemotherapy (Expert opinion).

\section{Chemotherapy}

\section{First-line systemic chemotherapy}

Docetaxel

Docetaxel $75 \mathrm{mg} / \mathrm{m}^{2}$ intravenous (IV) every three weeks with $\mathbf{5} \mathbf{~ m g}$ oral prednisone twice daily is recommended for patients with mCRPC (Level 1, Strong recommendation).

The TAX-327 study randomized 1006 patients to one of three treatment arms: 1 ) docetaxel $75 \mathrm{mg} / \mathrm{m}^{2}$ IV every three weeks; 2) docetaxel $30 \mathrm{mg} / \mathrm{m}^{2}$ weekly for five of six weeks; or 3) control therapy with mitoxantrone. ${ }^{19}$ The study reported improved survival with docetaxel (every three weeks) compared with mitoxantrone-prednisone (median survival 18.9 vs. 16.5 months; HR 0.76 ; $95 \%$ Cl 0.62-0.94; twosided $\mathrm{p}=0.009$ ). No OS benefit was observed with docetaxel given on a weekly schedule (HR 0.91; 95\% Cl 0.75-1.11; two-sided $p=0.36$ ). Significantly, more patients treated with docetaxel (every three weeks) achieved a pain response compared with patients receiving mitoxantrone $(35 \%$ vs. $22 \% ; p=0.01)$. Quality of life response, defined as a sustained 16-point or greater improvement from baseline on 
two consecutive measurements, was higher with docetaxel given every three weeks ( $22 \%$ vs. $13 \% ; p=0.009)$ or weekly ( $23 \%$ vs. $13 \%$; $p=0.005$ ) compared with mitoxantrone. PSA response rates were also statistically significantly higher with docetaxel compared to mitoxantrone. ${ }^{19}$ Although patients received up to 10 cycles of treatment if no progression and no prohibitive toxicities were noted, the duration of therapy should be based on the assessment of benefit and toxicities. Rising PSA alone should not be used as the sole criteria for progression; assessment of response should incorporate clinical and radiographic criteria.

Alternative therapies that have not demonstrated improvement in OS but can provide disease control, palliation, and improve quality of life include weekly docetaxel plus prednisone, and mitoxantrone plus prednisone (Level 2, Weak recommendation).

The timing of docetaxel therapy in men with evidence of metastases but without symptoms should be discussed with patients, and therapy should be individualized based on patients' clinical status and preferences (Level 3, Weak recommendation).

Patients who do not respond to first-line ADT or who progress clinically or radiologically without significant PSA elevations may have neuroendocrine differentiation. Biopsy of accessible lesions should be considered to identify these patients; these patients should then be treated with combination chemotherapy, such as cisplatin/etoposide or carboplatin/etoposide (Level 3, Weak recommendation).

Second-line systemic chemotherapy

Cabazitaxel

Cabazitaxel is recommended for $\mathrm{mCRPC}$ patients progressing on or following docetaxel (Level 1, Strong recommendation).

A phase 3 study comparing cabazitaxel to mitoxantrone in patients previously treated with docetaxel has shown a statistically significant survival advantage.$^{20}$ This randomized, placebo-controlled trial recruited 755 docetaxel-pretreated CRPC patients. OS was the primary endpoint of the study. Patients were randomized to receive prednisone $10 \mathrm{mg} /$ day with three times weekly mitoxantrone $12 \mathrm{mg} / \mathrm{m}^{2}$ or cabazitaxel $25 \mathrm{mg} / \mathrm{m}^{2}$. An advantage in survival emerged in favor of the cabazitaxel group, with a median survival of 15.1 months compared with 12.7 months in the mitoxantrone group (HR 0.70; 95\% Cl 0.59, 0.83; $\mathrm{p}<0.0001$ ). ${ }^{20}$

A recent phase 3 study comparing cabazitaxel $25 \mathrm{mg} / \mathrm{m}^{2}$ vs. $20 \mathrm{mg} / \mathrm{m}^{2}$ resulted in non-inferiority for cabazitaxel 20 $\mathrm{mg} / \mathrm{m}^{2}$ with less adverse events. Of note, in the subgroup analysis of patients who had received both docetaxel and abiraterone/enzalutamide, results appeared to favor a higher dose of cabazitaxel. ${ }^{21}$
Other options

For patients who have had a good response to first-line docetaxel, re-treatment with docetaxel can be considered (Expert opinion, Weak recommendation). ${ }^{22,23}$

Mitoxantrone has not shown any survival advantage but may provide symptomatic relief. Mitoxantrone may be considered a therapeutic option in symptomatic patients with $\mathbf{m C R P C}$ in the first- or second-line setting (Expert opinion, Weak recommendation).

\section{Bone-targeted therapy}

Life-prolonging therapy

\section{Radium-223}

Radium-223 every four weeks for six cycles is recommended in patients with pain due to bone metastases and who do not have visceral metastases (Level 1, Strong recommendation).

Radium-223 (previously known as alpharadin) is an intravenous alpha-emitting agent that mimics calcium, preferentially targeting bone metastases. In a randomized, phase 3 study, radium-223 given every four weeks for six cycles was compared to placebo. ${ }^{20}$ Radium-223 demonstrated a significant improvement in OS and symptomatic SREs. OS was improved by 3.6 months (HR 0.7; $\mathrm{p}<0.0001)$ and symptomatic SREs were delayed by 5.8 months $(p<0.0001)$. The study included patients with symptomatic bone metastases who were post-docetaxel or ineligible for docetaxel. ${ }^{24}$ The study excluded patients with visceral metastases or lymph node metastases greater than $3 \mathrm{~cm}$. PSA measurements while receiving radium-223 cannot provide evidence of whether patients are benefitting or not. Given the mechanism of action of the drug, alkaline phosphatase appears to be better marker of activity. A phase 3 study in the first-line mCRPC setting compared radium-223 in combination with abiraterone/prednisone vs. abiraterone/prednisone alone and demonstrated no advantage and an increased risk of fractures. ${ }^{25}$

Radium-223 should not be combined with abiraterone. A bone-supportive agent (denosumab or zoledronic acid) should always be used when using radium-223 (Level 1, Strong recommendation).

Patients with homologous recombination repair (HRR) mutations

Olaparib

Olaparib $300 \mathrm{mg}$ twice daily is recommended for patients with $\mathrm{mCPRC}$ and HRR mutation who have progressed on a previous androgen receptor-axis-targeted therapy (ARAT) (Level 1, Strong recommendation). 
HRR gene mutations occur in approximately $20-30 \%$ of prostate cancers from patients with metastatic disease, with the most common altered gene being BRCA2. Defective HRR renders a cancer susceptible to poly (ADP-ribose) polymerase (PARP) inhibition in a form of synthetic lethality. A randomized, phase 3 trial (PROfound) compared the PARP inhibitor, olaparib 300 mg BID, with physician's choice enzalutamide/abiraterone in patients with $\mathrm{mCRPC}$ with HRR mutations. Patients with HRR mutations and progression on prior enzalutamide and/or abiraterone with or without prior exposure to a taxane (docetaxel, cabazitaxel) were eligible. The primary endpoint of the study was radiographic PFS in patients with BRCA $1 / 2$ or ATM mutations. Results favored olaparib (7.39 vs. 3.44 months [HR 0.34, 95\% Cl (0.25, 0.47 $\mathrm{p}<0.001)$. The final results for OS also demonstrated a significant improvement among men with BRCA1/2 or ATM mutations, with a median OS of 19.1 vs. 14.7 months (HR 0.69, $95 \% \mathrm{Cl} 0.50,0.97, \mathrm{p}=0.02$ ). Of note, from patients in the physician's choice of enzalutamide/abiraterone arm who progressed, $67 \%$ crossed over to receive olaparib. Adjusting for crossover results in a HR $0.42(95 \% \mathrm{Cl} 0.19,0.91)$. Other key secondary endpoints include significant improvements in overall measurable response rates of $33.3 \%$ vs. $2.3 \%$ (odds ratio [OR] 20.86, $95 \% \mathrm{Cl} 4.18,379.18, \mathrm{p}<0.001)$ and delay in pain progression (HR 0.44, 95\% Cl 0.22, 0.91, p=0.0192). Adverse events were more common in the olaparib arm (anemia, fatigue, nausea, diarrhea), however, patients reported health-related quality of life was improved in the olaparib arm of the study.

The Health Canada approval of olaparib is for patients with deleterious or suspected deleterious germline or somatic BRCA1/2 or ATM mutations who have progressed following prior treatment with an NHT (i.e., abiraterone, enzalutamide, apalutamide, darolutamide). The U.S. Food and Drug Administration has approved olaparib for prostate cancers harboring a broader spectrum of 11 additional genes that are directly or indirectly involved in HRR (BRIP1, BARD1, CDK12, CHEK1, CHEK2, FANCL, PALB2, RAD51B, RAD51C, RAD51D, and RAD54L), which comprised an additional cohort in the PROfound study. The European regulatory authority has approved olaparib only for BRCA $1 / 2$ alterations. Further study is required to define optimal biomarker selection criteria to select patients with mCRPC with the highest potential for benefit from PARP inhibitors, as well as timing around taxane chemotherapy.

\section{Supportive agents}

Denosumab and zoledronic acid

In men with CRPC and bone metastases, denosumab (120 mg subcutaneous $[\mathrm{SC}]$ ) or zoledronic acid (4 mg IV) every four weeks are recommended to prevent disease-related
SREs, including pathological fractures, spinal cord compression, surgery, or radiation therapy to bone (Level 1 , Strong recommendation).

Bone loss associated with ADT has been shown to increase the risk of fracture. ${ }^{26-30}$ Moreover, about $90 \%$ of patients with mCRPC will develop bone metastases, which cause local decreases in bone integrity. Patients are at significant risk of SREs that include pathological fractures, debilitating bone pain requiring palliative radiation therapy, and spinal cord compression. Quality of life is affected by these complications.

Zoledronic acid is a third-generation nitrogen containing bisphosphonate. Bisphosphonates other than zoledronic acid are not known to be effective to prevent disease-related SREs. In the placebo-controlled zoledronic acid study, fewer men receiving zoledronic acid had SREs (38\% vs. $49 \%$; $\mathrm{p}=0.02){ }^{31}$ Zoledronic acid also increased the median time to first SRE (488 vs. 321 days; $p=0.01$ ). There was an overall $36 \%$ reduction in the rate of SREs in treated patients. ${ }^{31}$ Treatment with zoledronic acid should not be used in men with baseline creatinine clearance $<30 \mathrm{~mL} / \mathrm{min}$.

Denosumab is a fully humanized monoclonal antibody against RANK ligand. It has been shown to be effective in preventing bone loss and new vertebral fractures due to $\mathrm{ADT} .{ }^{30}$ In the setting of mCRPC, denosumab (120 mg SC every four weeks) compared to zoledronic acid (4 mg IV every four weeks) has shown significant improvement in the time to the first SRE (20.7 vs. 17.1 months; $p<0.001$ for non-inferiority; $p=0.008$ for superiority), while OS and PFS were not different. ${ }^{32}$

No dose modification for renal function is necessary in the case of denosumab; however, the risk of hypocalcemia is increased and calcium monitoring and supplementation (with calcium and vitamin D) is recommended for both denosumab and zoledronic acid. Denosumab has not been studied, however, in patients with severe renal impairment (glomerular filtration rate $<30 \mathrm{ml} / \mathrm{min}$ ).

Good oral hygiene, baseline dental evaluation for high-risk individuals, and avoidance of invasive dental surgery during therapy are recommended to reduce risk of osteonecrosis of the jaw (ONJ) for patients treated with bone-targeted therapies (Expert opinion). Zoledronic acid and denosumab have been used in combination with all the agents presently in use for the treatment of mCRPC. To date, there have been no additional safety issues of concern that have been reported.

The optimal duration of zoledronic acid and denosumab in men with CRPC and bone metastases is undefined. The risk of $\mathrm{ONJ}$ appears to be related to time on bone-targeted therapy, therefore, caution should be taken in using these agents beyond two years (Level 3, Weak recommendation).

Denosumab and zoledronic acid are not approved and not indicated for SRE prevention in the treatment of metastatic castration-sensitive prostate cancer or for bone metastases prevention. 


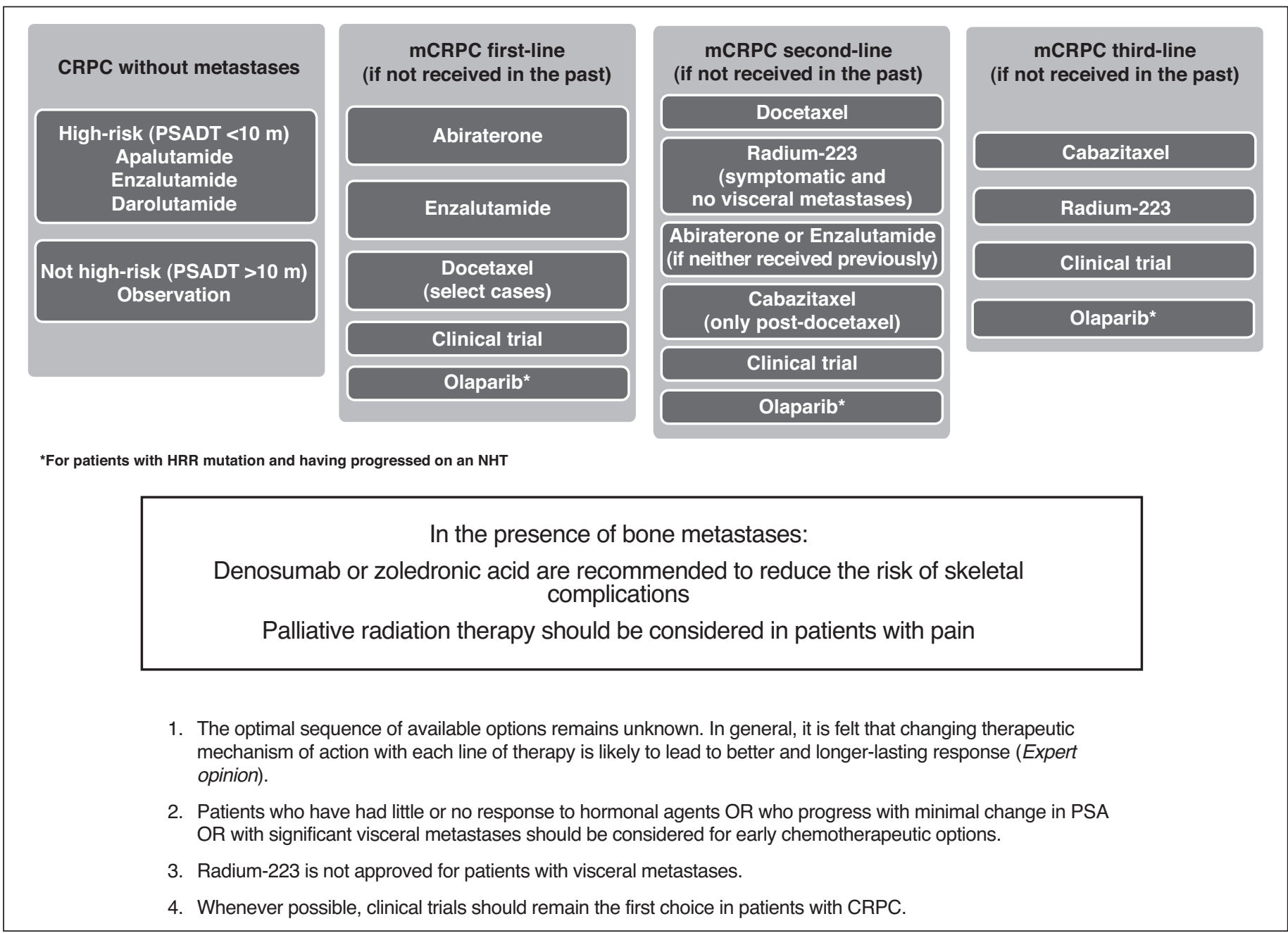

Fig. 1. Management of castration-resistant prostate cancer (CRPC). m: months; mCRPC: metastatic CRPC; HRR: homologous recombination repair; PSADT: prostate-specific antigen doubling time.

\section{Summary}

Health Canada-approved agents that have shown improvements in survival in $\mathrm{mCRPC}$ now include abiraterone, enzalutamide, docetaxel, cabazitaxel, and radium-223. Health Canada-approved agents that have shown improvements in delaying metastases in high-risk nmCRPC include apalutamide, enzalutamide, and darolutamide. Bone-supportive agents and palliative radiation continue to play an important role in the overall management of mCRPC. Given the complexity, variety, and importance of optimizing the use of these agents, a multidisciplinary team approach is highly recommended.

\section{Other supportive care therapies}

\section{Systemic corticosteroid therapy}

Corticosteroid therapy with low-dose prednisone or dexamethasone may also offer improvements in PSA values and/ or palliative outcomes in up to $30 \%$ of patients in both symptomatic and asymptomatic men. Steroids may also exert an anti-neoplastic effect on prostate cancer (Level 3, Weak recommendation). ${ }^{33}$

\section{Palliative radiation}

Bone metastases from prostate cancer are often radiosensitive and most men will experience partial or complete pain relief from external beam radiation to a specific lesion. ${ }^{34}$ Studies have shown that a single fraction of standard palliative radiotherapy (RT) is as effective as five or more fractions in providing palliation. However, more patients require retreatment for pain recurrence with single fraction radia- 
tion. Stereotactic body RT (SBRT) is a more precise and may be a more effective form of palliation delivered in five or fewer treatments and may also be considered, particularly for oligometastatic disease where high-dose RT is currently being studied for improved oncological outcomes.

Malignant spinal cord compression is an oncological emergency that requires immediate diagnosis with an MRI if suspected. Options for treatment are debulking surgery + RT, vertebrectomy with stabilization and RT, or RT + steroids (Level 1, Strong recommendation).

\section{Conclusions}

Advances in treatment for men with CRPC have improved survival and quality of life, but most, if not all, patients eventually succumb to their disease and better treatments are required. Several new agents are being studied in all states of CRPC and an increase in options is likely in the near future. Because CRPC remains an incurable and ultimately fatal illness, inclusion of patients in clinical trials remains paramount.

A summary on the recommended treatment of CRPC is shown in Fig. 1.

Competing interests: Dr. Saad has been an advisory board member for and has received payment/ honoraria from Abbvie, Amgen, Astellas, Bayer, Janssen, and Sanofi; and has participated in clinical trials supported by Amgen, Astellas, Bayer, Janssen, and Sanofi. Dr. Aprikian has been an advisory board member for Abbvie, Astellas, and Bayer; and has received grants from Abbvie, Astellas Bayer, Sanofi, and TerSera. Dr. Finelli has been an advisory board member for Abbvie, Astellas, Bayer, Janssen, Ipsen, Sanofi, and TerSera; and has participated in clinical trials supported by Astellas, Bayer, and Janssen. Dr. Fleshner has been a consultant or advisory board member for Abbvie, Amgen, Astellas, Bayer, Ferring, Hybridyne Health, Janssen, and Sanofi; and has participated in clinical trials supported by Astellas, Bavarian Nordic, Bayer, Ferring, Janssen, Medivalion, Nucleix, Progenics Pharmaceutical, Sanofi, and Spectracure AB. Dr. Gleave has been a consultant for and has received honoraria/grants from Abbvie, Amgen, Astellas, Bayer, GDx, Janssen, MDX, Pfizer, and Sanofi; and is founder/holds a patent with OncoGenex. Dr. Kapoor has been an advisory board member for BMS, Eisai, Ipsen, Merck, Novartis, Pfizer, and Roche; a speakers' bureau member for Eisai, Ipsen, Novartis, and Roche; and has received grants/honoraria from BMS, Eisai, Ipsen, Merck, Novartis, Pfizer, and Roche. Dr. Niazi has received research grants and honoraria from Abbvie, Amgen, Astellas, Astra Zeneca, Bayer, Janssen, and Sanofi; and has participated in clinical trials supported by Astellas, Ferring, Janssen, and Sanofi. Dr. North has been an advisory board member for Astellas, has received honoraria from Astra Zeneca, Astellas, Janssen, Merck, Roche, and Sanofi; and has participated in clinical trials supported by Astra Zeneca, Merck, Roche, and Sanofi. Dr. Pouliot has been an advisory board member for Amgen, Astellas, Bayer, and Janssen; has received payment from Abbott, Amgen, Astellas, Astra Zeneca, Bayer, Ferring, Janssen, and Sanofi; has received grants from Astra Zeneca and Sanofi; and has participated in clinical trials supported by Astellas, Bayer, Ferring, and Janssen. Dr. Rendon has been an advisory board and speakers' bureau member for and has received honoraria from Abbvie, Amgen, Astellas, Astra Zeneca, Bayer, Ferring, Jansen, and Sanofi. Dr. Shayegan has been an advisory board member for Astellas, Bayer, and Janssen; and has received a research grant from Janssen. Dr. Sridhar has been an advisory board member for Astellas, AstraZeneca, Bayer, Janssen, Merck, and Roche; and has participated in several pharma-supported clinical trials. Dr. So has been an advisory board member for Abbvie, Amgen, Astellas, Bayer, Janssen, Ferring, and TerSera; and has participated in clinical trials supported by Astellas, Ferring, and Janssen. Dr. Usmani has been an advisory board member for Amgen, Astellas, and Bayer; and has received grants from Best Medical and Concure Oncology Canada. Dr. Vigneault has been an advisory board member for Abbvie, Bayer, Ferring, and Sanofi. Dr. Chi has received honoraria from Astellas, Bayer, Janssen, and Sanofi; and has participated in clinical trials supported by Astellas, Astra Zeneca, Bayer, Eli Lilly, Essa, Janssen, Merck, Novartis, Pfizer, Roche, and Sanofi.
Prior to original publication, this guideline underwent review by the CUA Guidelines Committee, CUA members at large, the CUAJ Editorial Board, and the CUA Executive Board. 2020 updates were approved by the CUA Guidelines Committee.

\section{References}

1. Mohler JL, Gregory CW, Ford OH, 3rd, et al. The androgen axis in recurrent prostate cancer. Clin Cancer Res 2004;10:440-8. http://dx.doi.org/10.1158/1078-0432.CCR-1146-03

2. Scher HI, Halabi S, Tannock I, et al. Design and end points of clinical trials for patients with progressive prostate cancer and castrate levels of testosterone: recommendations of the Prostate Cancer Clinical Trials Working Group. J Clin Oncol 2008;26:1148-59. http://dx.doi.org/10.1200/JC0.2007.12.4487

3. Smith MR, Kabbinavar F, Saad F, et al. Natural history of rising serum prostate-specific antigen in men with castrate non-metastatic prostate cancer. J Clin Oncol 2005;23:2918-25. http://dx.doi.org/10.1200/ $J C 0.2005 .01 .529$

4. Small EJ, Halabi S, Dawson NA, et al. Antiandrogen withdrawal alone or in combination with ketoconazole in androgen-independent prostate cancer patients: A phase 3 trial (CALGB 9583). J Clin Oncol 2004;22:1025-33. http://dx.doi.org/10.1200/JC0.2004.06.037

5. Smith MR, Saad F, Chowdhury S, et al; SPARTAN Investigators. Apalutamide treatment and metastasis-free survival in prostate cancer. N Engl J Med 2018;378:1408-18. https://doi.org/10.1056/NEJMoal715546

6. Hussain M, Fizazi K, Saad F, et al. Enzalutamide in men with non-metastatic, castration-resistant prostate cancer. N Engl J Med 2018;378:2465-74. https://doi.org/10.1056/NEJMoal 800536

7. Fizazi K, Shore N, Tammela TL, et al; ARAMIS Investigators. Darolutamide in non-metastatic, castrationresistant prostate cancer. N Engl J Med 2019 Feb 14. [Epub ahead of print]. https://doi.org/10.1056/ NEJMoa1815671

8. Saad F, Cella D, Basch E, et al. Effect of apalutamide on health-related quality of life in patients with nonmetastatic castration-resistant prostate cancer: An analysis of the SPARTAN randomized, placebo-controlled, phase 3 trial. Lancet Oncol 2018;19:1404-16. https://doi.org/10.1016/S1470-2045(18)30456-X

9. Smith MR, Saad F, Chowdhury S, et al. Apalutamide and overall survival in prostate cancer. Eur Urol 2020. Epub ahead of print. https://doi.org/10.1016/.eururo.2020.08.011

10. Tombal B, Saad F, Penson D, et al. Patientreported outcomes following enzalutamide or placebo in men with non-metastatic, castration-resistant prostate cancer (PROSPER): A multicenter, randomized, double-blind, phase 3 trial. Lancet Oncol 2019 Feb 12. [Epub ahead of print]. https://doi.org/10.1016/S1470-2045(18)30898-2

11. Sternberg CN, Fizazi K, Saad F, et al; PROSPER Investigators. Enzalutamide and survival in non-metastatic, castration-resistant prostate cancer. N Engl J Med 2020;382:2197-2206. https://doi.org/10.1056/ NEJMoa2003892

12. Fizazi K, Shore N, Tammela TL, et al; ARAMIS Investigators. Non-metastatic, castration-resistant prostate cancer and survival with darolutamide. N Engl J Med 2020;383:1040-9. hitps://doi.org/10.1056/ NEJMoa2001342

13. Ryan CJ, Smith MR, de Bono IS, et al. Abiraterone in metastatic prostate cancer without previous chemotherapy. N Engl J Med 2013;368:138-48. https://doi.org/10.1056/NEJMoal 209096

14. Ryan CJ, Smith MR, Fizazi K, et al. Abiraterone acetate plus prednisone vs. placebo plus prednisone in chemotherapy-naive men with metastatic castration-resistant prostate cancer (COU-AA-302): Final overall survival analysis of a randomized, double-blind, placebo-controlled phase 3 study. Lancet Oncol 2015;16: 152-60. https://doi.org/10.1016/S1470-2045(14)71205-7

15. de Bono JS, Logothetis CJ, Molina A, et al. Abiraterone and increased survival in metastatic prostate cancer. N Engl J Med 2011; 364:1995-2005. https://doi.org/10.1056/NEJMoa1014618

16. Beer TM, Armstrong AJ, Rathkopf DE, et al. Enzalutamide in metastatic prostate cancer before chemotherapy. N Engl J Med 2014;371:424-33. http://dx.doi.org/10.1056/NEJMoal 405095

17. Beer TM, Armstrong AJ, Rathkopf $D$, et al. Enzalutamide in men with chemotherapy-naive metastatic castration-resistant prostate cancer: Extended analysis of the phase 3 PREVAll study. Eur Urol 2017;71: 151-4. https://doi.org/10.1016/i.eururo.2016.07.032

18. Scher HI, Fizazi K, Saad F, et al. Increased survival with enzalutamide in prostate cancer after chemotherapy. N Engl J Med 2012;367:1187-97. http://dx.doi.org/10.1056/NEJMoal207506

19. Tannock IF, de Wit R, Berry WR, et al. Docetaxel plus prednisone or mitoxantrone plus prednisone for advanced prostate cancer. N Engl J Med 2004;351:1502-12. http://dx.doi.org/10.1056/NEJMoa040720

20. de Bono IS, Oudard $S$, Ozguroglu $M$, et al. Prednisone plus cabazitaxel or mitoxantrone for metastatic castration-resistant prostate cancer progressing after docetaxel treatment: A randomized open-label trial. Lancet 2010;376:1147. http://dx.doi.org/10.1016/S0140-6736(10)61389-X

21. Eisenberger M, Hardy-Bessard AC, Kim CS, et al. Phase 3 study comparing a reduced dose of cabazitaxel ( $20 \mathrm{mg} /$ $\left.\mathrm{m}^{2}\right)$ and the currently approved dose $\left(25 \mathrm{mg} / \mathrm{m}^{2}\right)$ in post-docetaxel patients with metastatic castration-resistant prostate cancer-PROSELLCA. J Clin Oncol 2017;35:3198-3206. https://doi.org/10.1200/JC0.2016.72.1076 
22. Jankovic B, Beardsley E, Chi KN. Rechallenge with docetaxel as second-line chemotherapy in patients with metastatic hormone refractory prostate cancer (HRPC) after previous docetaxel: A population-based analysis [abstract \#196]. 2008 ASCO Genitourinary Symposium; 2008.

23. Eymard J, Oudard $S$, Gravis $G$, et al. Docetaxel reintroduction in patients with metastatic castration-resistant docetaxel-sensitive prostate cancer: A retrospective, multicentre study. BJU Int 2010;106:974-8. http://dx.doi.org/10.1111/i.1464-410X.2010.09296.x

24. Parker C, Nilsson S, Heinrich D, et al. Alpha emitter radium-223 and survival in metastatic prostate cancer. N Engl J Med 2013;369:213-23. http://dx.doi.org/10.1056/NEJMoal213755

25. Smith M, Parker C, Saad F, et al. Addition of radium-223 to abiraterone acetate and prednisone or prednisolone in patients with castration-resistant prostate cancer and bone metastases (ERA 223): A randomized, double-blind, placebo-controlled, phase 3 trial. Lancet Oncol 2019 Feb 6. [Epub ahead of print]. https://doi.org/10.1016/S1470-2045(18)30860-X

26. Diamond TH, Higano CS, Smith MR, et al. Osteoporosis in men with prostate carcinoma receiving androgendeprivation therapy: Recommendations for diagnosis and therapies. Cancer 2004;100:892. http:// dx.doi.org/10.1002/cncr.20056

27. Shahinian VB, Kuo YF, Freeman JL, et al. Risk of fracture after androgen deprivation for prostate cancer N Engl J Med 2005;352:154-64. http://dx.doi.org/10.1056/NEJMoa041943

28. Smith MR, McGovern FJ, Zietman AL, et al. Pamidronate to prevent bone loss during androgen-deprivation therapy for prostate cancer. N Engl J Med 2001;345:948-55. http://dx.doi.org/10.1056/NEJMo0010845

29. Smith MR, Eastham J, Gleason DM, et al. Randomized controlled trial of zoledronic acid to prevent bone loss in men receiving androgen-deprivation therapy for non-metastatic prostate cancer. J Urol 2003; 169:200812. http://dx.doi.org/10.1097/01.ju.0000063820.94994.95
30. Smith MR, Egerdie B, Hernandez Toriz N, et al. Denosumab in men receiving androgen-deprivation therapy for prostate cancer. N Engl J Med 2009;361:745-55. http://dx.doi.org/10.1056/NEJMoa0809003

31. Saad F, Gleason DM, Murray R, et al. Long-term efficacy of zoledronic acid for the prevention of skeletal complications in patients with metastatic hormone refractory prostate cancer. J Natl Cancer Inst 2004;96:879-82. http://dx.doi.org/10.1093/inci/dih141

32. Fizazi K, Carducci $M$, Smith $M$, et al. Denosumab vs. zoledronic acid for treatment of bone metastases in men with castration-resistant prostate cancer: A randomized, double-blind study. Lancet 2011;377:813. http://dx.doi.org/10.1016/S0140-6736(10)62344-6.

33. Storlie JA, Buckner JC, Wiseman GA, et al. Prostate-specific antigen levels and clinical response to lowdose dexamethasone for hormone-refractory metastatic prostate carcinoma. Cancer 1995;76:96-100. http://dx.doi.org/10.1002/1097-0142(19950701)76:1<96::AID-CNCR2820760114>3.0.C0;2-E

34. Loblaw DA, Mitera G, Ford $M$, et al. A 2011 updated systematic review and clinical practice guideline for the management of malignant extradural spinal cord compression. Int J Radiat Oncol Biol Phys 2012;84:312-7. http://dx.doi.org/10.1016/i.irobp.2012.01.014.

Correspondence: Dr. Fred Saad, Centre Hospitalier de I'Université de Montréal, Montreal, QC, Canada; fredsaad@videotron.ca 


\section{CUA-CUOG CRPC guideline summary}

Castration-resistant prostate cancer (CRPC) includes a wide range of disease types: from patients without metastases or symptoms with rising prostate-specific antigen (PSA) levels despite androgen deprivation therapy (ADT) to patients with metastases and significant debilitation due to cancer symptoms.

\section{Androgen deprivation therapy}

Because androgen receptor remains active in most patients who have developed castration-resistant disease, it is recommended that ADT be continued for the remainder of a patient's life (Strong recommendation).

\section{Rising PSA non-metastatic CRPC}

1. ADT should be maintained in the nmCRPC state (Level 3, Strong recommendation). First-generation androgen receptor antagonists (i.e., bicalutamide, flutamide, etc.) should be discontinued if patients are receiving these agents (Level 3, Weak recommendation).

2. Men with high-risk nmCRPC, defined as a PSADT $<10$ months, with an estimated life expectancy of greater than five years should be offered apalutamide, enzalutamide, or darolutamide (Level 1, Strong recommendation).

3. In men with high-risk nmCRPC who are felt to be unsuitable or refuse approved therapies, observation or use of first-generation androgen receptor antagonists may be attempted (Level 3, Weak recommendation).

4. Men with nmCRPC who are not considered high-risk, observation or secondary hormonal treatments may be attempted (Level 3 , Weak recommendation).

5. Patients who are untreated for nmCRPC should be followed with regular imaging every 6-12 months depending on PSA doubling time (PSADT) (Level 3, Weak recommendation).

II. Chemotherapy-naive metastatic CRPC (MCRPC) without symptoms or minimally symptomatic

1. Abiraterone acetate $1000 \mathrm{mg} /$ day plus prednisone $5 \mathrm{mg} /$ twice daily is recommended as first-line therapy (Leve/ 1 , Strong recommendation).

2. Enzalutamide $160 \mathrm{mg} /$ day is recommended as first-line therapy (Level 1, Strong recommendation).

3. Docetaxel $75 \mathrm{mg} / \mathrm{m}^{2}$ every three weeks plus $5 \mathrm{mg}$ oral prednisone twice daily can be offered (Level 1, Strong recommendation). The timing of docetaxel therapy in men with evidence of metastases but without symptoms should be discussed with the patient and therapy should be individualized based on the patient's clinical status and preference.

III. mCRPC with moderate or severe symptoms

1. Docetaxel $75 \mathrm{mg} / \mathrm{m}^{2}$ every three weeks plus $5 \mathrm{mg}$ oral prednisone twice daily is recommended (Level 1, Strong recommendation).

2. Radium-223 every four weeks for six cycles is recommended in patients with pain due to bone metastases and who do not have visceral metastases (Level 1, Strong recommendation). Radium-223 significantly improved overall survival and reduced symptomatic skeletal-related events in patients with symptomatic mCRPC who had previously received docetaxel chemotherapy or were deemed unfit for docetaxel.

3. Abiraterone acetate $1000 \mathrm{mg} /$ day plus prednisone $\mathbf{5} \mathbf{~ m g}$ twice daily or enzalutamide $\mathbf{1 6 0} \mathrm{mg} /$ day may be considered as first-line therapy in patients who cannot receive or refuse docetaxel (Expert opinion).

IV. mCRPC who progress after docetaxel-based chemotherapy

Options with survival benefit

1. Cabazitaxel (25 mg/m²) plus prednisone (5 $\mathbf{~ m g / d a y ) ~ ( L e v e l ~ 1 , ~ S t r o n g ~ r e c o m m e n d a t i o n ) . ~}$

2. Radium-223 every four weeks for six cycles (Level 1, Strong recommendation).

3. If not received prior to docetaxel:

i. Abiraterone acetate (1000 $\mathbf{~ m g}$ per day) plus prednisone (5 $\mathbf{~ m g}$ twice daily) (Level 1, Strong recommendation)

ii. Enzalutamide (160 $\mathbf{m g} /$ day) (Level 1, Strong recommendation)

Options with unknown survival benefit

1. Docetaxel plus prednisone re-exposure in patients who have had a previous favorable response to docetaxel may be reasonable (Expert opinion).

2. Mitoxantrone plus prednisone may be offered for palliative pain relief (Expert opinion, Weak recommendation).

V. Patients with CRPC and bone metastases (includes the pre- or post-chemotherapy settings)

1. Denosumab (120 $\mathrm{mg}$ subcutaneous) or zoledronic acid (4 $\mathrm{mg}$ intravenous) every four weeks, along with daily calcium and vitamin $D$ supplementation is recommended to prevent disease-related skeletal complications (Level 1, Strong recommendation).

VI. Patients with mCPRC and HRR mutation who have progressed on a previous ARAT with or without taxane exposure

1. Olaparib $300 \mathrm{mg} \mathrm{BID}$ 